\title{
Philosophiques
}

\section{Qui a peur des qualia corporels?}

\section{Jérôme Dokic}

Volume 27, numéro 1, printemps 2000

Le matérialisme contemporain

URI : https://id.erudit.org/iderudit/004917ar

DOI : https://doi.org/10.7202/004917ar

Aller au sommaire du numéro

\section{Éditeur(s)}

Société de philosophie du Québec

ISSN

0316-2923 (imprimé)

1492-1391 (numérique)

Découvrir la revue

\section{Citer cet article}

Dokic, J. (2000). Qui a peur des qualia corporels? Philosophiques, 27(1), 77-98. https://doi.org/10.7202/004917ar

\section{Résumé de l'article}

Les qualia , considérés comme des propriétés intrinsèques de nos expériences, ne sont pas toujours bien accueillis par les matérialistes, qui préfèrent les considérer comme des propriétés intentionnelles présentées dans l'expérience. Je me demande si cette forme de réductionnisme s'applique aux qualia de la conscience corporelle. Selon la théorie matérialiste standard, l'objet intentionnel de l'expérience de la douleur, par exemple, est un dommage physique. Mais cette théorie ne rend pas compte de la différence phénoménale entre ressentir une douleur " de l'intérieur » et la percevoir « à l'extérieur ». J'esquisse une autre analyse réductionniste, compatible avec le matérialisme, selon laquelle l'objet intentionnel de la conscience corporelle, contrairement à celui de la conscience externe, dépend de manière constitutive de l'expérience du sujet.
Ce document est protégé par la loi sur le droit d'auteur. L'utilisation des services d'Érudit (y compris la reproduction) est assujettie à sa politique d'utilisation que vous pouvez consulter en ligne.

https://apropos.erudit.org/fr/usagers/politique-dutilisation/ 


\title{
Qui a peur des qualia corporels?
}

\author{
JÉRÔME DOKIC \\ dokic@poly.polytechnique.fr \\ Université de Rouen et CREA, École polytechnique
}

\begin{abstract}
RÉSUMÉ. - Les qualia, considérés comme des propriétés intrinsèques de nos expériences, ne sont pas toujours bien accueillis par les matérialistes, qui préfèrent les considérer comme des propriétés intentionnelles présentées dans l'expérience. Je me demande si cette forme de réductionnisme s'applique aux qualia de la conscience corporelle. Selon la théorie matérialiste standard, l'objet intentionnel de l'expérience de la douleur, par exemple, est un dommage physique. Mais cette théorie ne rend pas compte de la différence phénoménale entre ressentir une douleur « de l'intérieur » et la percevoir « à l'extérieur ». J'esquisse une autre analyse réductionniste, compatible avec le matérialisme, selon laquelle l'objet intentionnel de la conscience corporelle, contrairement à celui de la conscience externe, dépend de manière constitutive de l'expérience du sujet.
\end{abstract}

\begin{abstract}
Qualia, conceived as intrinsic properties of experiences, are not always welcome by materialists, who prefer to see them as intentional properties presented in our experience. I ask whether this form of reductionism applies to qualia of bodily awareness. According to the standard materialist theory, the intentional object of pain experience, for instance, is a bodily damage. This theory, though, is unable to account for the phenomenal difference between feeling pain " from inside" and perceiving it "outside" (seeing oneself or another in pain). I sketch another reductionist analysis which is compatible with materialism, and according to which the intentional object of bodily awareness, unlike that of external perception, constitutively depends on the subject's experience.
\end{abstract}

\section{Les qualia et la conscience intentionnelle}

«Pour un matérialiste, aucun fait n'est accessible qu'à une seule personne ${ }^{1}$ ». Et pourtant, certaines de nos expériences - principalement celles qui relèvent de la conscience perceptive et de la conscience corporelle - semblent avoir une qualité intrinsèque, un « caractère phénoménal » souvent considéré comme étant essentiellement privé. Thomas $\mathrm{N}$ agel (ou plutôt ses traducteurs) parle d'un « effet que cela fait » d'avoir une expérience de ce type ${ }^{2}$. Cet « effet » est déterminé par les qualia associés à notre expérience, auxquels il semble que nous ayons immédiatement accès par introspection.

Plutôt que d'éliminer les qualia, qui sont profondément enracinés dans notre vie consciente telle que nous la concevons, certains philosophes préconisent de renoncer à la thèse selon laquelle ils sont des propriétés phénoménales intrinsèques de nos expériences ${ }^{3}$. Fred Dretske écrit à propos de la

1. Dretske, Fred, $N$ aturalizing the $M$ ind, Cambridge (M ass.), M IT Press, 1995, p. 65.

2. N agel, Thomas, "Q uel effet cela fait-il d'être une chauve-souris? », dans Q uestions mortelles, traduction P. Engel et C. Engel-Tiercelin, Paris, PUF, 1983.

3. Selon une autre option, l'existence de qualia considérés comme des propriétés intrinsèques de l'expérience est compatible avec une vision matérialiste ou naturaliste du monde ; 
perception externe que « la qualité de l'expérience, la manière dont les choses nous apparaissent au niveau sensoriel, est constituée par les propriétés de celles-ci telles qu'elles nous sont représentées ${ }^{4} \gg$. Ce qui nous apparaît comme des qualités de l'expérience sont en réalité des propriétés présentées dans l'expérience. Comme ces propriétés sont typiquement extérieures au sujet et à ses états mentaux, le programme qui consiste à réduire les qualia à des propriétés ou à des aspects de la scène objective perçue repose sur une forme d'externalisme. II s'agit en fait d'une forme assez radicale d'externalisme, puisque toutes les propriétés de l'expérience immédiatement accessibles à la conscience sont attribuées à la scène objective perçue. Comme le dit Robert Kirk, c'est un programme « ultra-externaliste », puisqu'il a pour but de « donner une explication philosophique de tous les aspects de la conscience uniquement sur la base du contenu des états mentaux déterminé de manière externe $e^{5} »$.

La réussite d'un tel programme est soumise à plusieurs conditions. Elle dépend avant tout de la possibilité d'une théorie matérialiste ou naturaliste del'intentionnalité6. Uneautre condition concernela nature des objets intentionnels eux-mêmes. La réduction des qualia à des propriétés présentées dans l'expérience ne sert le matérial isme que dans la mesure où celles-ci sont ellesmêmes objectives et naturelles. Or on a affirmé l'existence de qualités phénoménal es représentées comme étant instanciées par les objets eux-mêmes de la scène perçue, et non directement par l'expérience. Ces qualités seraient pourtant constituées, en définitive, par des relations entre ces objets et le sujet conscient, éventuellement par l'entremise de propriétés intrinsèques de son expérience ${ }^{7}$.

voir par exemple Shoemaker, Sydney, The First-Person Perspective and O ther Essays, Cambridge, Cambridge University Press, 1996, p. 264. Shoemaker rejette également l'idée selon laquelle les qualia sont directement accessibles à la conscience; ils sont connus pour ainsi dire par description. Un doute est alors permis : Shoemaker rend-il vraiment justice à la notion intuitive de qualia?

4. Dretske, $N$ aturalizing the $M$ ind, p. 1.

5. Kirk, R obert, Raw Feeling, Oxford, Clarendon Press, 1994, p. 54. H ormis D retske, d'autres représentants de ce programme (ou d'une variante plus ou moins radicale de celui-ci) incluent $\mathrm{H}$ arman, Gilbert, " The Intrinsic Q uality of Experience », dans Tomberlin, James, dir., Philosophical Perspectives, vol. 4, N orthridge (Calif.), Ridgeview, 1990 ; Tye, M ichael, Ten Problems of Consciousness. A Representational Theory of the Phenomenal $\mathrm{M}$ ind, Cambridge (M ass.), M IT Press, 1995 et Lycan, William, Consciousness and Experience, Cambridge (M ass.), M IT Press, 1996.

6. Il y a certes des différences importantes entre ce qui est matériel, ce qui est physique et ce qui est naturel, mais elles ne jouent pas un rôle substantiel dans cet essai.

7. Voir Shoemaker, The First-Person Perspective, qui réintroduit de cette manière la notion de qualia. Pour une autre conception des qualités phénoménales appliquée à la perception externe, qui ne revendique pas l'existence de propriétés intrinsèques de l'expérience, voir M CD owell, J ohn, "Values and Secondary Q ualities », dans H onderich, Ted, dir., M orality and O bjectivity, Boston, Routledge and Kegan Paul, 1985, p. 110-129. 
Je laisserai de côté, dans cet essai, le problème de la naturalisation de I'intentionnalité, pour me concentrer sur celui de la réduction des qualia corporels à des propriétés intentionnelles (des propriétés présentées dans l'expérience). Par « qualia corporels », j'entends les qualia associés à la conscience du corps propre, c'est-à-dire à l'expérience directe que nous avons, " de l'intérieur », de notre propre corps et des conditions dans lesquelles il se trouve. Lorsque j'ai mal à la jambe, ou que je ressens un picotement dans le bras, j'ai conscience qu'une partie de mon corps se trouve dans une certaine condition qui m'est ainsi directement accessible. Le cas de la conscience corporelle est particulièrement important pour le défenseur du réductionnisme. Premièrement, cette forme de conscience est incontestablement associée à des qualia, à tel point que le langage ordinaire reconnaît plus volontiers l'existence des sensations corporelles que celle des sensations visuelles, auditives ou olfactives. D euxièmement, la thèse sel on laquelle la conscience corporelle a une dimension intentionnelle est plus controversée que dans le cas de la perception externe ; par exemple, certains philosophes considèrent la douleur comme une simple sensation dénuée de signification intentionnelle $e^{8}$. Enfin, à supposer que la conscience corporelle soit effectivement une expérience intentionnelle, il reste à montrer que ses objets intentionnels font partie du mobilier du monde matériel.

À mes yeux, la réduction des qualia de la perception externe à des propriétés intentionnelles est une stratégie prometteuse. Je tenterai néanmoins de montrer que la conscience corporelle pose des problèmes spécifiques que certains auteurs ont largement sous-estimés. Les conceptions matérialistes actuelles de la conscience corporelle sont soit très incomplètes, soit contraires à l'expérience. Je brosserai à grands traits une analyse de la conscience corporelle qui est en principe compatible avec les aspirations réductionnistes et naturalistes. L'intérêt d'une telle discussion est double. D'une part, elle indique que la conscience corporelle n'est pas un obstacle infranchissable pour qui se réclame du programme matérialiste. D'autre part, elle ouvre la voie à une analyse plus réaliste de la conscience corporelle qui, je l'espère, a un intérêt philosophique intrinsèque.

\section{L'objectivité de la conscience corporelle}

La conscience corporelle a-t-elle un objet intentionnel? Est-elle une forme de perception objective? Si c'est le cas, la perception consciente est un genre qui comporte (au moins) deux espèces : la conscience externe (la vision, le toucher, l'ouïe, etc.) et la conscience corporelle. II y aurait donc une essence de la perception plus générale que celle de la perception externe, et qui définit également la conscience corporelle. On peut commencer par dégager trois

8. Voir Searle, John, «Intentionality (1)», dans Guttenplan, Samuel, dir., A Companion to the Philosophy of M ind, Oxford, Blackwell, 1994, p. 380. 
traits fondamentaux qui participent clairement de l'essence de la perception externe ${ }^{9}$, et qui semblent s'appliquer aussi à la conscience corporelle :

1. Une condition minimale de la perception objective concerne la localisation spatiale. Concevoir une expérience objective, c'est avant tout concevoir une expérience spatiale ${ }^{10}$. J e peux en principe localiser les objets de la conscience visuelle sur la seule base de mon expérience. N ormalement, les objets de la conscience corporelle sont localisés dans le corps propre, ressentis comme ayant une certaine épaisseur spatiale. L'objet de mon expérience corporelle peut être localisé de manière essentiellement vague, comme lorsque je ressens une douleur diffuse dans l'ensemble du corps, mais il est souvent localisé assez précisément, comme lorsque je viens de me piquer le doigt avec une aiguille à coudre. De même qu'il est difficile d'imaginer un son qui ne vienne de nulle part, il est difficile d'imaginer une sensation corporelle qui n'ait aucune signification spatiale.

C'est parce que l'objet de la conscience corporelle se présente comme étant localisé que certains phénomènes caractéristiques de la perception objective sont possibles. Par exemple, je peux avoir une douleur à la main gauche et une autre, exactement similaire, à la main droite; la distinction entre deux douleurs qualitativement similaires mais numériquement distinctes est rendue intelligible par la dimension spatiale de la conscience corporelle. Le rapport constitutif des sensations corporelles à l'espace nous permet également d'envisager une douleur qui se déplace le long du bras ou, pourquoi pas, deux douleurs qualitativement différentes qui se « croisent » sur la surface du corps sans se confondre.

2. Une autre notion fondamentale liée à la perception objective est celle d'attention. II y a sans doute une notion d'attention spécifique à l'expérience de type perceptif, par opposition à la pensée consciente ${ }^{11}$. J e peux porter mon attention successivement sur différentes régions de mon champ visuel. (Le concept d'attention et celui de champ perceptif sont d'ailleurs étroitement liés.) D e même, je peux porter mon attention sur la douleur que je ressens au pied droit, puis sur celle que je ressens à la main gauche. Par ailleurs, porter son attention visuelle sur un objet permet typiquement de recueillir davan-

9. Shoemaker, The First-Person Perspective, p. 204-206, propose une liste de huit traits constitutifs de la perception sensorielle (sense-perception). Le premier trait mentionné ici ne figure pas explicitement dans sa liste, mais il permet sans doute, avec celui qui concerne l'attention, d'expliquer d'autres traits moins fondamentaux. Par exemple, la perception objective permet de prendre conscience de faits parce qu'elle est avant tout une conscience d'objets (trait 3 chez Shoemaker) ; elle permet aussi d'avoir des informations qui identifient l'objet de la perception (trait 4).

10. Voir Strawson, Peter F., L es individus, traduction A. Shalom et P. D rong, Paris, Seuil, 1973, ch. 2, et Evans, Gareth, "Things Without the M ind », dans Collected Papers, Oxford, Oxford University Press, 1985.

11. Voir Peacocke, Christopher, "Conscious Attitudes, Attention, and SelfKnowledge », dans Wright, Crispin, Smith, Barry C. et M acdonald, Cynthia, Knowing O ur O wn M ind, Oxford, Oxford University Press, 1998. 
tage d'informations sur lui. Il en va de même pour la conscience corporelle. Je peux me concentrer sur ma douleur et lui découvrir, sur la base de mon expérience attentive, de nouvelles propriétés.

3. On pose souvent comme principe que là où il y a perception objective, il doit y avoir la possibilité d'illusions ou d'hallucinations. Si je suis capable de percevoir une table réelle devant moi, je peux également être victime d'une illusion : il n'y a aucune table devant moi (hallucination) ou je perçois la table comme ayant des propriétés qu'elle n'a pas réellement (illusion $)^{12}$. Il est vrai que la notion d'illusion ou d'hallucination de la conscience corporelle n'est pas solidement ancrée dans l'usage ordinaire, mais nous sommes au moins enclins à l'utiliser jusqu'à un certain point. Par exemple, le sujet victime d'une douleur « référée » croit ressentir la douleur dans son bras alors qu'elle concerne en réalité son cœur. Le patient qui, après une amputation, a des sensations dans sa jambe fantôme a une hallucination. Enfin, je peux corriger mon jugement à l'égard d'une sensation corporelle je pensais qu'il s'agissait d'une sensation de pression, et je me rends compte que c'est en fait une douleur légère ${ }^{13}$.

Je supposerai ici que ces traits doivent être pris au sérieux, et qu'il est difficile d'en rendre compte simplement en invoquant la thèse suspecte sel on laquelle nous « projetons » dans l'espace des sensations corporelles dénuées de signification spatiale. Toutefois, certains philosophes acceptent la thèse sel on laquelle la conscience corporelle est spatiale, mais refusent de la considérer comme une forme de perception objective. Pour la tradition phénoménologique, la conscience corporelle ne présente pas au sujet un état de choses objectif, à la manière de la perception externe. Si la douleur, par exemple, m'est présentée dans un " espace douloureux ${ }^{14}$ », elle n'est pas un « objet » dont je pourrais avoir l'expérience à partir de plusieurs points de vue différents, comme lorsque jevariel'angle sous lequel j'observe une table. C'est toujours et nécessairement à partir du même point de vue que je fais l'expérience de ma douleur (celui de mon propre corps), ce qui indiquerait que l'on ne peut pas parler, au sens strict, de perception objective ${ }^{15}$.

12. Cette distinction entre illusion et hallucination vient de Austin, John L., L e langage dela perception, traduction P. G ochet, Paris, A rmand Colin, 1971. Le principe qui lie la capacité de perception véridique à la possibilité d'être victime d'une illusion est compatible avec la " théorie disjonctive de la perception », selon laquelle un acte particulier de perception véridique n'aurait pas pu être illusoire (voir H inton, James, Experiences, Oxford, Clarendon Press, 1973).

13. Voir Armstrong, David, Bodily Sensations, London, Routledge and Kegan Paul, 1962, p. 53, et Pitcher, George, «Pain Perception », Philosophical Review, 79, 1970, p. 388.

14. Voir M erleau-Ponty, Maurice, La phénoménologie de la perception, Paris, Gallimard, 1945.

15. La thèse selon laquelle la conscience corporelle n'est pas une forme de perception objective va de pair avec le refus de considérer le corps propre comme un « objet », qui pourrait entrer et sortir du champ de la conscience comme les objets de la perception externe. 
Du point de vue matérialiste, toutefois, la position phénoménologique est une pétition de principe. Si l'on peut montrer que l'objet de la conscience corporelle est de même nature que celui de la perception externe, la même chose pourra en principe être perçue de deux «points de vue » différents. Considérons l'expérience de la douleur. Selon la Théorie M atérialiste Standard (TM S), l'objet intentionnel de cette expérience est un dommage ou un désordre physique relatif à une partie de mon corps plus ou moins délimitée. Lorsque j'ai mal au bras, mon expérience me présente un tort corporel d'un certain type - mon bras est meurtri, blessé, irrité, dérangé. Comme le dit D retske, « de même que l'expérience visuelle d'un arbre est la conscience (awareness) d'un objet non conscient (l'arbre), la douleur est une conscience d'une condition corporelle non consciente (une partie blessée, foulée ou malade) ${ }^{16} »$. Des remarques analogues valent mutatis mutandis pour les autres sensations corporelles. La conscience corporelle a un objet intentionnel, mais celui-ci fait partie du domaine d'objets reconnu par le matérialiste.

La TM S peut être motivée par des arguments physiologiques. O n peut dire, avec Shoemaker, qu'un trait stéréotypique supplémentaire de la perception objective est le fait que « les croyances perceptives sont causalement produites par les objets ou les états de choses perçus, par l'entremise d'un mécanisme causal qui, normalement, produit des croyances vraies ${ }^{17} »$. Toute perception objective suppose l'existence d'un lien causal et informationnel entre l'expérience et son objet. Sur le plan neurophysiologique, les mécanismes qui sous-tendent l'expérience de la douleur véhiculent de l'information sur certains dommages corporels. Des terminaisons nerveuses, appelées « nocicepteurs», se trouvent dans la plupart des tissus corporels. Les nocicepteurs répondent de manière sélective à des stimuli nocifs - principalement aux déformations mécaniques, à l'augmentation de la chaleur et de la concentration de certaines substances chimiques ${ }^{18}$.

Selon la TM S, la conscience corporelle peut être considérée (Iorsqu'elle est véridique) comme une forme de perception spatiale d'un état de choses objectif qui concerne le corps propre. Cet état de choses - par exemple, un dommage physique - est en principe accessible à la perception externe. Toutefois, cette théorie se heurte à un obstacle majeur. II y a sans doute une différence notable ( $c$ 'est un euphémisme) entre percevoir un désordre physique « de l'intérieur », c'est-à-dire ressentir une douleur, et percevoir le même désordre « à l'extérieur », par le biais dela perception externe. Si les deux for-

16. Dretske, N aturalizing the $M$ ind, p. 102. Voir aussi Pitcher, "Pain Perception», $\mathrm{H}$ arman, «The Intrinsic Q uality of Experience », Tye, Ten Problems of Consciousness et Lycan, Consciousness and Experience. $O \mathrm{n}$ trouve des éléments de cette conception chez $\mathrm{R}$ eid, Thomas, Essays on the Intellectual Powers of $M$ an, edited and abridged by A. D. Woozley, Londres, M acmillan and Co, 1941, p. 165-169.

17. Shoemaker, The First-Person Perspective, p. 206.

18. Voir Fields, Howard L. et Price, Donald D., «Pain », dans Guttenplan, dir., A Companion to the Philosophy of M ind, p. 452-459. 
mes de conscience - corporelle et externe - peuvent partager leurs objets, elles ne présentent manifestement pas ceux-ci de la même manière. Le même état corporel objectif est présenté au sujet sous deux modes radicalement différents. D e tels modes de présentation ne sont assurément pas bienvenus pour un matérialiste qui préconise la réduction des qualia à des propriétés purement intentionnelles. II y aurait en effet des expériences qui porteraient sur la même scène objective perçue mais qui différeraient sur le plan phénoménal. La difficulté pour le matérialiste consiste alors à rendre compte de ces différences sans faire intervenir à nouveau des qualia corporels.

La concession qui consiste à accepter l'existence de modes de présentation perceptifs fait ressortir toute la force de la thèse phénoménologique traditionnelle : si la conscience corporelle et la conscience externe présentent la réalité sous des modes radicalement différents, il est naturel de soupçonner qu'en définitive, les objets intentionnels de ces deux formes de conscience ne relèvent pas de la même catégorie ontologique ${ }^{19}$. N ous ne sommes pas loin del'idée selon laquelle il y a un espace réservé aux sensations corporelles, qui n'est pas de même nature que l'espace dans lequel sont perçus les objets de la perception externe ${ }^{20}$.

L'un des buts de cet article est d'essayer de tracer une voie intermédiaire entre la position de la phénoménologie traditionnelle, assez coûteuse sur le plan ontologique, et la conception matérialiste standard, qui parvient difficilement à expliquer les différences phénoménales entre la conscience corporelle et la conscience externe. Je vais commencer par préciser le sens dans lequel l'objet de la conscience corporelle est présenté d'une manière radicalement différente de celui de la conscience externe.

\section{Un modèle discutable de la conscience corporelle}

Le problème central dela TM S consisteà rendre compte de la différence intuitive qui existe entre percevoir un dommage physique « de l'intérieur », c'està-dire ressentir une douleur, et percevoir le même dommage « à l'extérieur »,

19. On peut ainsi lever l'objection selon laquelle l'existence de modes de présentation perceptifs distincts des objets intentionnels de l'expérience ne représente aucune difficulté pour le matérialiste, en tout cas pas plus quel'existence de termes co-référentiels qui ont des sens (non descriptifs) différents. Certes, si le contenu de l'expérience est entièrement conceptuel (voir M cD ow ell, John, M ind and World, Cambridge (M ass.), H arvard University Press, 1994), il n'y a aucune différence de nature entre les deux types de modes de présentation - sur le plan perceptif et sur celui de la pensée conceptuelle. Cette objection ne tient pas dans le cas spécifique de la conscience corporelle. Comme nous le verrons dès la section suivante, les différences entre celle-ci et la conscience externe sont telles qu'il est particulièrement difficile de maintenir la thèse selon laquelle le même état de choses est présenté sous deux modes différents, encore moins si ces modes sont purement conceptuels.

20. Sur cepoint, M erleau-Ponty est clair : la distinction entre ces deux genres d'espace est tracée à l'intérieur même de l'expérience. C'est une distinction phénoménologique, alors que la notion d'espace physique est d'ordre scientifique et vient de la réflexion. 
par exemple par la vue. Cette différence est liée au fait que l'espace de la douleur ressentie, contrairement à celui des objets vus, a des bornes relativement bien définies. Tout d'abord, la douleur telleque jela ressens concerne toujours une partie corporelle, jamais une chaise ou un cendrier. M ais, deuxièmement, elle n'est pas localisée dans n'importe quelle partie corporelle; elle concerne toujours mon corps. Je ne peux pas ressentir une douleur dans un corps qui ne m'appartient pas. Toute douleur que je ressens est localisée dans mon espace corporel.

Le cas du membre fantôme semble constituer à cet égard une exception. Le sujet qui a mal à son bras fantôme ne ressent-il pas une douleur en un lieu extérieur peut-être vide? M ême dans ce cas, pourtant, le sujet a l'impression (illusoire) de ressentir la douleur dans une partie du corps qui n'existe plus. La douleur est ressentie comme étant localisée dans son espace corporel apparent : le sujet ne se débarrasse pas de sa douleur en quittant la pièce ; la douleur le « suit » dans tous ses déplacements ${ }^{21}$.

Le défenseur de la TM S peut tenter d'affirmer qu'il est contingent, et non pas nécessaire, que la douleur soit toujours ressentie dans l'espace corporel. Il invoquera peut-être W ittgenstein, qui semble avoir sérieusement envisagé la possibilité de ressentir véritablement une douleur dans la main d'autrui, ou même dans un meuble $e^{22}$. Un certain modèle de la conscience corporelle paraîtra alors inévitable. C'est un fait queles nocicepteurs, qui jouent un rôle crucial dans l'expérience de la douleur, se trouvent à l'intérieur ou à la surface du corps, mais il aurait pu en aller autrement. De même que certains procédés artificiels repoussent les limites du champ visuel (une paire de lunettes ou de jumelles), on peut imaginer une prothèse qui prolongerait l'expérience de la douleur au-delà de l'espace corporel. De ce point de vue, la conscience corporelle est essentiellement une forme de perception externe ; elle est seulement beaucoup plus «myope», pour ainsi dire, que la vision. Comme la lumière, qui est le milieu de l'information relative à l'objet de la perception visuelle, le système nerveux serait une sorte de milieu de l'information relative au dommage corporel perçu.

Les nerfs eux-mêmes sont remplaçables. O n peut imaginer, par exemple, que les nocicepteurs qui se trouvent dans la main de mon voisin sont branchés à un émetteur (en plus d'être rattachés normalement au cerveau de ce dernier). Cet émetteur transmet des informations à un récepteur situé dans mon corps, qui lui-mêmeles fait parvenir à mon cerveau. Si une abeilles'avise

21. Voir Langsam, H ., «W hy Pains are M ental O bjects », Journal of Philosophy, 92 (6), 1995, p. 304.

22. Voir Wittgenstein, Ludwig, Le cahier bleu et le cahier brun, traduction G. Durand, Paris, Gallimard, 1965, p. 100 et suivantes. II va sans dire que W ittgenstein n'est pas matérialiste au sens présupposé ici. II ne considère pas non plus dans ce texte l'expérience de la douleur comme une forme de perception d'un dommage corporel. II est davantage intéressé par les conditions (fictives) dans lesquelles nous serions amenés à localiser une sensation de douleur dans le corps d'autrui, et parler par exemple du «mal de dent à la dent d'un tel ». 
de piquer la main de mon voisin, et si l'information fournie par les nocicepteurs et retransmise à mon cerveau est préservée (autrement dit, si le « bruit » informationnel est réduit au minimum), je ressentirai comme mon voisin une douleur déterminée. Certes, pour rendre cescénario plausible, il faut invoquer un critère d'identité substantiel du corps propre qui soit indépendant de la conscience corporelle. N ous disposons d'un contre-exemple à l'intuition selon laquelle la douleur ressentie est intrinsèquement liée au corps propre seulement s'il est indépendamment établi que la main de mon voisin ne fait pas partie de mon corps (au moment où je ressens la douleur en question).

L'intention du matérialiste est ici de montrer que les qualia corporels apparemment responsables de l'effet que cela fait de ressentir la douleur « de l'intérieur » ne sont pas des propriétés essentielles del'expérience corporelle. Cela revient à éliminer les qualia de ce genre s'il prétend en plus que le contraste intuitif qui existe entre la conscience externe et la conscience corporelle est illusoire.

La situation est en réalité plus complexe, puisqu'il faut distinguer deux cas de figure. Dans le premier cas, lorsque je ressens une douleur dans la main de mon voisin, je n'ai pas conscience que cette main fasse partie de mon corps propre ; j'ai même conscience qu'elle n'en fait pas partie. A utrement dit, il y aurait une dissociation entre la conscience des sensations corporelles éventuellement localisées dans l'espace extracorporel et la conscience « de l'intérieur » des limites spatiales de mon corps propre, qui serait indépendante des sensations corporelles. Dans le deuxième cas de figure, lorsqu'il me semble ressentir la douleur dans la main de mon voisin, j'ai également conscience que celle-ci fait partie de mon corps propre : j'ai l'impression d'avoir une troisième main. Pourtant, ma conscience « de l'intérieur » des limites de mon corps propre, contrairement à mon expérience de la douleur, est illusoire ; en réal ité, la douleur que je ressens concerne un corps qui ne m'appartient pas. Dans ce cas aussi, il y a une dissociation entre la conscience des sensations corporelles et celle du corps propre, puisque les deux types d'expérience n'ont pas les mêmes conditions de satisfaction. Toutefois, à la différence du premier cas, cette dissociation n'est pas apparente sur le plan phénoménologique. A ucun de ces cas de figure n'est favorable au matérialiste, puisqu'ils reviennent tous deux à créditer la conscience « del'intérieur » des limites du corps propre des qualia qui ont été refusés à la conscience corporelle.

En résumé, il n'est pas aisé d'éliminer les qualia corporels responsables del'effet que cela fait de ressentir une sensation « de l'intérieur ». Ces qualia semblent être essentiels à la conscience corporelle, et participer de l'impression que nous avons des limites de notre corps propre.

\section{Un critère d'identité partiel du corps propre}

Le matérialiste n'est pas obligé de prendre au sérieux le modèle de la conscience corporelle esquissé dans la section précédente ; il peut tenter de rendre 
justice à l'intuition selon laquelle la douleur ressentie est intrinsèquement liée à l'espace corporel. Les limites de la conscience corporelle sont par définition celles du corps propre. II est certes possible que nos nocicepteurs se trouvent en dehors de ce qui est de facto notre corps. $M$ ais lorsque nous envisageons cette possibilité, nous ne considérons pas que la douleur est ressentie dans l'espace extra-corporel ; nous supposons au contraire que les limites de cet espace se sont déplacées, et quelepoint auquel nous ressentons la douleur (dans la situation envisagée) fait maintenant partie intégrante de notre corps. Comme le dit A rmstrong, " c'est un fait contingent que les qualités sensibles [telles que la douleur] concernent les objets qu'elles concernent et pas d'autres, mais cen'est pas un fait contingent que ces objets sont appelés les corps des personnes ${ }^{23} »$.

Selon la définition envisagée, une condition suffisante pour qu'un membre donné appartienne au corps propre d'un sujet est que celui-ci ressente ou pourrait ressentir une sensation corporelle localisée dans ce membre. Ce ne peut pas être une condition nécessaire de la possession du corps propre, du moins si nous admettons que nous avons beaucoup de parties corporelles dont nous ne sommes normalement pas conscients. En continuant à prendre la douleur comme paradigme de la sensation corporelle, nous obtenons le critère d'identité partiel suivant :

(D) Si j'ai mal (ou si je pouvais avoir mal) à $x$, alors $x$ fait partie de mon corps propre, ou inclut une limite de celui-ci.

Une difficulté immédiate liée à ce critère concerne les conditions de satisfaction de la conscience corporelle. II ne s'agit pas d'affirmer que si je ressens apparemment une douleur en un endroit déterminé de l'espace, celuici est occupé par une partie de mon corps propre. Une telle affirmation conduirait à la définition d'un corps purement phénoménal (éventuellement constitué en partie de membres fantômes), qui ne pourrait être identifié à aucun objet matériel. De plus, l'objet intentionnel de l'expérience de la douleur ne pourrait plus être identifié dans tous les cas à un dommage physique. Sel on une lecture de (D) plus acceptable pour le matérial iste, si je ressens véritablement une douleur en un endroit déterminé de l'espace, celui-ci est occupé par une partie de mon corps propre. Le problème consiste maintenant à définir ce qui compte comme une expérience corporelle véridique. La simple existence d'un lien causal/informationnel entre un dommage physique et une expérience corporelle ne suffit pas à rendre celle-ci véridique. En I'absence d'un critère de véridicité de la conscience corporelle, le critère (D) apparaîtra entièrement ad hoc au philosophe qui, comme Wittgenstein, admet la possibilité de ressentir véritablement la douleur dans un corps étranger. Comment distinguer le cas possible où je localise de manière illusoire la douleur dans un corps étranger de celui où mon corps propre incorpore (temporairement) la main d'autrui? 
II est intéressant de comparer le critère (D) à ce que Q uassim Cassam appelle «l'idéalisme concernant la possession du corps ${ }^{24}$ ». Selon cette position, attribuée à Locke, notre corps est entièrement défini comme celui dont nous avons conscience de la manière appropriée, c'est-à-dire « de l'intérieur ». Une partie corporelle appartient au corps du sujet si et seulement si le sujet ressent ou peut ressentir des sensations corporelles dans cette partie. Les sensations corporelles constituent le seul critère adéquat d'identité du corps propre.

Le critère (D) n'est pas idéaliste au sens de Cassam, avant tout parce qu'il ne fait pas de la conscience corporelle une condition nécessaire de l'appartenance d'un membre donné au corps propre du sujet. M ais considéronsl'objection que Cassam soulève contre l'idée sel on laquelle la conscience corporelle est une condition suffisante de la possession du corps. R evenons au scénario dans lequel la main de mon voisin est branchée à mon cerveau par l'entremise d'ondes radio. Supposons que je ressente une douleur que je localise, sur la seule base de mon expérience, dans cette main. Selon l'idéalisme concernant la possession du corps, il s'ensuit que la main appartient à mon corps propre. Selon Cassam, une telle conclusion est inacceptable car « elle ne respecte pas l'intuition selon laquelle un membre auquel on n'est pas matériellement uni ne peut pas faire partie de soi $\mathrm{i}^{25} \gg$. Le principe invoqué est ici que «I'on ne peut pas être matériellement uni à un membre spatialement éloigné, auquel on n'est pas localement rattaché26 ».

Pour rendre justice à cette intuition, Cassam oppose à l'idéalisme une position qu'il appelle « réaliste », selon laquelle « il est nécessaire et suffisant, pour qu'un membre ou une partie corporelle fasse partie de nous, que ce membre ou cette partie soit matériellement uni à nou ${ }^{27}$ ». Bien entendu, le réalisme concernant la possession du corps soulève immédiatement la question de savoir ce qui fait qu'un corps donné, matériellement uni, constitue notre corps. M ais je laisserai ce point de côté. Ce qui m'intéresse, c'est la possibilité pour le matérialiste de restreindre les conditions de véridicité de l'expérience de la douleur à celles où l'objet intentionnel est un dommage physique relatif à un corps matériellement uni. Le défenseur de la TM S pourrait donc affirmer, en invoquant le réalisme au sens de Cassam, qu'une expé rience corporelle est illusoire si le dommage physique avec lequel elle a un lien causal/informationnel concerne une partie matériellement distincte du reste du corps. Cette nouvelle définition exclut du domaine des expériences corporelles véridiques la conscience du sujet dans le scénario wittgensteinien.

La stratégie matérialiste dépend ici du bien-fondé de la position que Cassam qualifie de « réaliste ». Le réal isme exclut la possi bilité que les parties de notre corps propre soient éparpillées dans le monde. Or il n'est pas du tout

24. Cassam, Q uassim, Self and World, O xford, Clarendon Press, 1997.

25. Ibid., p. 65.

26. Ibid.

27. Ibid., p. 66. 
évident que cette possibilité soit toujours problématique, comme semble l'affirmer Cassam. M ême si cette possibilité n'est en fait jamais réalisée, nous pouvons nous imaginer sans incohérence qu'elle le soit. Supposons par exemple que nous puissions volontairement détacher notre main de notre bras pour explorer tactilement une région de l'espace qui nous est autrement inaccessible. (II faut imaginer que la main possède son propre mécanisme de locomotion.) Sans melever ni déranger mes compagnons, je pourrais « détacher » ma main et aller chercher la salière se trouvant à l'autre bout de la table ${ }^{28}$.

Lescénario qui vient d'être évoqué est curieux, mais pas contradictoire. De manière générale, il n'y a aucune absurdité conceptuelle dans l'idée que le sujet peut agir, peut-être par télékinésie, en des points disjoints de l'espace. À cet égard, il est intéressant de relever que la psychologie expérimentale reconnaît l'existence de modules perceptivo-moteurs spécialisés. Les traitements spatiaux requis pour l'action sont fragmentés en multiples systèmes de contrôle relativement indépendants entre eux. Par exemple, différentes informations perceptives et différents groupes moteurs sont impliqués lorsque le bras se déplace vers un objet, et lorsque les doigts le saisissent ${ }^{29}$.

Il est vrai que notre conscience spatiale, corporelle et externe, est normalement unifiée. L'unité de l'expérience découle directement de l'exigence générale sel on laquelle le sujet doit pouvoir localiser l'objet perçu sur la seule base de son expérience. Pour que le sujet puisse percevoir un objet comme étant à un endroit déterminé de l'espace, il doit pouvoir le localiser relativement à tous les autres objets qu'il perçoit au même moment, y compris le corps et ses différentes parties. Le champ perceptif est un système de relations spatiales synesthésiques, c'est-à-dire entre objets qui peuvent être perçus au travers de différentes modalités sensorielles ${ }^{30}$.

Sel on le matérialisme, la conscience corporelle est une forme de perception objective. $M$ ais pour que ma main détachée puisse contribuer à mon expérience globale du monde, les données qu'elle fait apparaître doivent être orientées par rapport au reste des données sensorielles dont j'ai l'expérience. L'orientation relative est une condition nécessaire pour que je puisse placer les informations véhiculées par mon expérience dans un espace dans lequel je me trouve également, et donc pour queje puisse me servir de ma main pour percevoir le monde qui m'entoure.

28. Sur la main détachable, voir aussi Casati, R oberto et Dokic, Jérôme, La philosophie du son, N îmes, Éditions Chambon, 1994.

29. Voir Rossetti, Yves, "Des modalités sensorielles aux représentations spatiales en action : représentations multiples d'un espace unique », dans Proust, Joëlle, dir., Perception et intermodalité. A pproches actuelles de la question de M olyneux, Paris, PUF, 1997. Je ne veux pas exclure qu'il y ait des actions qui engagent l'ensemble du corps, comme le déplacement d'un endroit à l'autre, ni que ces actions jouent un rôle important dans la constitution de l'objectivité de notre expérience.

30. Voir D okic, Jérôme, «La signification des expressions égocentriques », dans Proust, dir., Perception et intermodalité. 
En d'autres termes, ma main reste intégrée au reste de mon corps seulement si elle est spatialement reliée à celui-ci dans mon champ perceptif global. Tant que je garde la trace perceptive de l'orientation de la main par rapport au reste de mon corps, j'ai une expérience corporelle bizarre peutêtre, mais unifiée. Je peux considérer ma main comme une partie spatialement disjointe de mon corps.

Q ue se passe-t-il si je perds la trace de l'orientation relative de la main? Ce serait le cas si, par exemple, ma main détachée disparaissait de mon champ visuel pendant un certain temps - si je quittais la pièce en oubliant de rattacher ma main au reste de mon corps. Ce cas serait similaire, sous certains aspects, à la situation dans laquelle se trouvent certains sujets qui ont perdu presque entièrement le toucher et la proprioception musculaire ${ }^{31}$. Ces patients peuvent ressentir la douleur et avoir certaines sensations thermiques, mais ils sont incapables de pointer du doigt l'endroit stimulé sur leur corps s'ils ne le voient pas également. Par contre, ils peuvent nommer l'endroit en question et le désigner sur une représentation schématique du corps, ce qui indique que leur image corporelle n'a pas entièrement disparu.

On peut observer que ces patients ne localisent pas le dommage corporel sur la seule base de leur expérience de la douleur. C ette expérience, contrairement à la nôtre, ne leur fait pas apparaître les relations spatiales synesthésiques qui existent entre la partie corporelle douloureuse et les parties corporelles visibles. Ils sont contraints de passer par une représentation anatomique de leur corps pour retrouver l'endroit douloureux dans leur champ de vision. De même, il est possible que je sois capable de nommer la partie douloureuse de ma main - que je sache que c'est l'index qui me fait mal, par exemple. M ais cela n'implique pas que mon expérience de la douleur soit véridique. Elle ne pourra pas l'être si je suis incapable de localiser l'index dans mon champ perceptif sur la seule base de mon expérience.

II est possible aussi que je garde l'impression que ma main est orientée d'une certaine façon relativement au reste de mon corps. A insi, j'ai l'impression de percevoir certaines données tactiles à un endroit déterminé dans mon champ perceptif global. $M$ ais cette impression ne peut pas être véridique si le lien nomologique entre l'orientation apparente et I'orientation véritable est rompu. M ême si l'orientation apparente de la main correspondait à son orientation véritable, ce serait un pur accident et la main ne serait pas véritablement perçue.

Une possibilité beaucoup plus spéculative, mais qui ne peut pas être écartée a priori, est que la main détachée devienne un autre corps propre, littéralement aveugle. J'aurais ainsi la capacité de « sauter » d'un corps dans

31. Cole, Jonathan et Paillard, Jacques, "Living without Touch and Peripheral Information about Body Position and M ovement : Studies with Deafferented Subjects », dans Bermúdez, J osé Luis, M arcel, Anthony et Eilan, $\mathrm{N}$ aomi, dir., The Body and the Self, Cambridge (M ass.), M IT Press, 1995. 
I'autre, et de me retrouver instantanément en des lieux complètement différents. II n'est pas nécessaire d'explorer cette possibilité plus avant. II suffit de souligner que les considérations qui précèdent ne portent pas contre la possibilité d'avoir différents corps à différents moments, mais plutôt contre la possibilité de jouir de deux points de vue simultanés sur le monde qui ne soient pas spatialement reliés l'un à l'autre dans un champ perceptif global. Le don d'ubiquité en ce sens n'existe pas ${ }^{32}$.

En résumé, une condition relative à l'unité de l'expérience doit effectivement être incluse dans les conditions de véridicité de la conscience corporelle (et donc dans la définition du corps propre), mais elle découle d'une condition plus générale qui concerne l'unité spatiale et synesthésique du champ perceptif. Corrélativement, elle n'implique pas directement l'unité matérielle du corps. On objectera que pour que les parties de mon corps puissent transmettre des informations à mon cerveau, il faut qu'elles soient liées entre elles au moins par une relation causale. Cette objection est sans doute correcte, mais elle montre seulement que nous concevons difficilement la possibilité d'une communication immédiate entre deux points disjoints dans l'espace, qui ne dépende pas d'une transmission causale de l'information d'un point à l'autre continûment. La difficulté conceptuelle que nous avons ici n'est pas spécifique au problème du corps propre.

\section{Un bref rappel des options}

Dans les deux dernières sections, j'ai soulevéun certain nombre de difficultés liées à la TM S, qui ont trait au contraste intuitif entre la conscience externe et la conscience corporelle. $\mathrm{O} n$ a distingué deux options. Selon la première, les sensations corporelles ne sont pas nécessairement localisées dans le corps propre. Comme nous l'avons vu, cette option ne permet pas d'éliminer les qualia corporels, qui réapparaissent associés à une forme de conscience du corps propre détachée des sensations corporelles (et dont l'existence est douteuse). La deuxième option, qui a l'avantage de respecter l'intuition ordinaire, consiste à définir le corps propre au moyen des sensations corporelles. $\mathrm{N}$ on seulement cette option est, nous l'avons vu, ad hoc, mais elle ne fait que mettre en évidence le problème qui se pose pour la TM S. Si l'objet intentionnel de la conscience douloureuse n'est qu'un simple dommage physique, il est légitime de se demander ce qu'elle a de spécial par rapport à la conscience externe du même dommage, en ce qu'elle définit les limites du corps propre. Le critère (D) ne fait que souligner le fossé qui existe entre ces deux formes de conscience. Si avoir mal, c'est percevoir un dommage corporel qui, par essence, n'est pas lié à un corps particulier, un tel critère apparaît comme étant tout à fait immotivé.

32. Voir Strawson, Peter F., The Bounds of Sense, Londres, R outledge and Kegan Paul, 1966, p. 150-152, et Evans, Gareth, The Varieties of R eference, O xford, Blackwell, 1982, ch. 6. 
Pour sortir de cette impasse, je suggère de renoncer à l'idée selon laquelle l'objet intentionnel de l'expérience de la douleur est un simple dommage physique. Dans le reste de cet essai, je vais procéder en trois étapes. Premièrement, je vais rappeler un argument selon lequel les sensations corporelles engagent de manière constitutive des réponses spontanées de la part du sujet. Deuxièmement, je vais montrer que ces réponses participent de l'objet intentionnel de la conscience corporelle. Enfin, je vais tenter de mettre en évidence la dimension réflexive de la conscience corporelle, qui selon moi contribue à expliquer la différence pertinente entre ressentir la douleur « de l'intérieur » et la percevoir « à l'extérieur ».

\section{Les conditions d'émergence de la conscience objective}

$L a$ thèse selon laquelle la conscience corporelle engage nécessairement des réponses spontanées est la conséquence d'un argument plus général présenté par D avid Pears (à partir de W ittgenstein) et avant lui par Friedrich H ayek ${ }^{33}$. $\mathrm{L}^{\prime}$ argument de $\mathrm{H}$ ayek/Pears ne concerne pas la conscience corporelle en particulier, mais toute forme d'expérience intentionnelle de type sensoriel ${ }^{34}$.

En discutant les objections de Wittgenstein contre la possibilité d'un langage privé, Pears affirme que « le langage doit se surimposer à un réseau préexistant de connexions allant des stimuli physiques jusqu'au seuil de la conscience, et en retour jusqu'au comportement ${ }^{35} »$. Lorsque l'enfant introduit dans son langage un nouveau terme qui désigne une sensation, il le fait toujours sur un arrière-plan de liens prélinguistiques complex es entre des stimulations sensorielles et des réponses motrices. Ces liens relèvent de ce que Pears appelle des « lignes de types ». Ces lignes de types sont solidement établies avant l'avènement du langage, et prédéterminent le sens et l'usage du terme de sensation.

L'existence de connexions prélinguistiques entre des stimuli et des réponses est particulièrement évidente dans le cas de la douleur. Chez une personne normale, les stimuli nocifs sont associés naturellement à un comportement de rejet approprié à la situation - retirer brusquement sa main d'un radiateur brûlant en est un exemple particulièrement visible. L'idée générale de Pears est quele type de douleur ressentie est déterminé par unelignequi va decertains stimuli à certaines réponses spontanées. Si jeveux introduire un termequi désigne un certain type de douleur, je dois tenir compte des deux moitiés de la sensation,

33. Q uel ques paragraphes de cette section et de la suivante sont tirés de mon article « $\mathrm{La}$ perception interne et la critique du langage privé », R evue de théologie et de philosophie, 130, 1998, p. 1-19. Je parviens ici à une conclusion similaire, mais par une voie un peu différente.

34. Un argument similaire, qui concerne la localisation dans le champ perceptif, a été présenté par Pitcher, G eorge, A Theory of Perception, Princeton, Princeton University Press, 1971 et Evans, The Varieties of R eference.

35. Pears, D avid, L a pensée-W ittgenstein : du Tractatus aux R echerches Philosophiques, traduction C. Chauviré, Paris, Aubier, 1993, p. 371. 
réceptrice et motrice. J e ne peux pas introduire un terme de sensation privée qui identifierait la douleur indépendamment de son expression naturelle ${ }^{36}$.

Suivant Pears, les qualités accessibles au sujet d'une expérience consciente sont différenciées les unes des autres par des réponses naturellement associées à certaines stimulations sensorielles. L'idée est que des éléments perçus qui diffèrent sur le plan phénoménal doivent correspondre à des réponses publiquement observables différentes. $D$ ans le cas précis, ces réponses déterminent non seulement le lieu, l'étendue et l'intensité de la douleur ressentie, mais préfigurent également son caractère intrinsèquement désagréable. Les réponses spontanées sont déjà des amorces de rejet de la partie corporelle stimulée, ou du moins des tentatives d'échapper à l'agression extérieure ${ }^{37}$.

H ayek présente un argument similaire à celui de Pears, sauf qu'il concerne non pas l'introduction d'un nouveau terme de sensation, mais l'émergence d'une expérience objective consciente. Selon lui, les stimuli sensoriels sont associés à des réactions spontanées, et cette association naturelle est antérieure à toute expérience objective. Bien que $\mathrm{H}$ ayek ne mentionne pas explicitement l'expérience de la douleur, ses remarques sont applicables à ce cas spécifique. Sur le plan physiologique, lorsquel'information véhiculée par les nocicepteurs parvient au système nerveux central, elle est déjà investie d'une certaine « signification » pour l'organisme. Le système nerveux central reçoit presque simultanément deux « rapports » concomitants : I'un concernant le stimulus nocif, l'autre ayant trait à la réaction spontanée du corps ${ }^{38}$.

Les lignes de types sont solidement établies avant I'avènement du langage (Pears) et l'émergence d'une expérience consciente (H ayek), mais des connexions régulières entre les stimuli nocifs et les réponses spontanées sont nécessaires pour maintenir à travers le temps la signification du terme de sensation et le contenu intentionnel de l'expérience ${ }^{39}$. Toutefois, les lignes de types acquièrent un statut différent lorsque le langage et l'expérience sont en place.

36. Voir Wittgenstein, Ludwig, Investigations philosophiques, traduction P. K lossowski, Paris, Gallimard, 1961, §245: « Comment puis-je aller jusqu'à vouloir intervenir au moyen du langage entre la douleur et son expression? »

37. Je suppose ici que l'expérience de la douleur est intrinsèquement désagréable indépendamment des attitudes émotionnelles qui lui sont typiquement associées. On peut contester cette supposition : des patients lobotomisés ou ayant pris de la morphine disent ressentir la même douleur avec la même intensité qu'avant l'opération ou la prise de la drogue, mais affirment qu'elle ne les gêne plus; leur expérience de douleur n'est plus désagréable, ce qui implique peut-être l'absence de réactions négatives spontanées (voir Dennett, Daniel, "W hy You Can't M ake a Computer that Feels Pain », dans Brainstorms, Cambridge (M ass.), M IT Press, 1978). On peut débattre de la question de savoir s'il faut prendre ou non les dires de ces patients à la lettre. Cette question est relativement indépendante de mon propos dans cet essai. M ême si l'expérience de douleur n'est pas intrinsèquement désagréable en ce sens, elle reste liée à des réponses spontanées qui déterminent le type de sensation ressentie - le lieu, l'étendue et l'intensité de la douleur.

38. H ayek, Friedrich, The Sensory O rder, Chicago, University of Chicago Press, 1953, p. $79-101$.

39. Voir Pears, L a pensée-W ittgenstein, p. 407. 
A u commencement, on ne trouve que des stimulations sensorielles et des réponses motrices dénuées de signification intentionnelle. $M$ ais les deux termes des lignes de types prennent une autre dimension avec l'avènement du langage et l'émergence de la conscience objective. D 'une part, le lien régulier entre un dommage corporel et le stimulus nocif est tel que l'expérience de douleur peut être considérée comme une forme de perception intentionnelle (confuse) du dommage. D'autre part, les réactions aux stimuli nocifs, bien qu'elles restent spontanées, acquièrent également un statut proto-intentionnel. Premièrement, elles peuvent être interprétées comme des réponses aux dommages euxmêmes comme stimuli « distaux » (bien que normalement à l'intérieur du corps), et non pas à des stimuli nocifs « proximaux » (autour des nocicepteurs). D euxièmement, chez l'individu développé, les connexions prélinguistiques entre stimuli et réponses sont suivies et orientées par des comportements supérieurs, plus directement contrôlés par lui. Ces comportements sont capables, dans une certaine mesure, d'inhiber ou de modifier les réponses directes dans le contexte plus général d'autres perceptions et intentions ${ }^{40}$. Le caractère proto-intentionnel des réponses est défini précisément par le fait qu'elles sont sensibles à un tel contexte. M ême si en un certain sens, elles ne sont pas entiè rement voulues par le sujet, elles représentent quel que chose que nous faisons naturellement, et pas seulement quelque chose qui est fait en nous. M ême les mouvements réfl exes sont typiquement influencés par la perception de la situation générale : je peux lâcher une tasse de café brûlante dans un contexte et la reposer maladroitement sur une table proche dans un autre ${ }^{41}$.

Lorsqu'il n'y a aucune réponse observable, comme dans le cas d'une douleur faible, les stimuli nocifs sont quand même associés par nature à une certaine tendance à réagir « visiblement » (par exemple par une estimation verbale de l'intensité de la douleur - mais d'autres manifestations sont possibles). Toutefois, cette tendance n'est pas une simple disposition ; elle constitue déjà un début d'activité réelle qui imprime une direction spécifique à tout comportement fondé sur l'expérience de la douleur. Dans tous les cas, il doit y avoir une certaine harmonie entre les réponses spontanées et les comportements motivés par des attitudes à l'égard de la douleur, telles que le désir qu'elle cesse immédiatement ${ }^{42}$. Précisément en raison du fait que ces attitudes sont fondées sur l'expérience consciente de la douleur plutôt qu'antérieures à celle-ci, elles en sont dissociables dans un contexte plus large où d'autres considérations interviennent - par exemple, une disposition au masochisme. M ême le masochiste, pourtant, ne peut pas aller entièrement contre sa nature, et doit résister aux réponses spontanées s'il ne les souhaite pas.

40. Voir Hayek, The Sensory O rder, p. 85.

41. Voir M erleau-Ponty, M aurice, La structure du comportement, Paris, PUF, 1942 ; M elzack, R. et Wall, P., The Challenge of Pain, London, Penguin Books, 1988 (deuxième édition), p. 193.

42. Voir Evans, The Varieties of Reference, p. 159. 


\section{Une définition des sensations corporelles}

Selon l'argument wittgensteinien de Pears, les règles sémantiques qui gouvernent l'usage de tous les termes de sensations - que celles-ci relèvent de la conscience externe ou de la conscience corporelle - sont édifiées sur un sol naturel de liens préréflexifs entre la perception et le comportement. II existe néanmoins une différence cruciale entre les deux formes de conscience, que I'on peut présenter de la manière suivante. II se trouve qu'un terme comme « rouge » désigne une propriété qui convient naturellement à la cause « distale » de notre expérience chromatique. Sa référence est (un aspect de) I'entrée de la ligne de type qui sous-tend son utilisation, même si son sens dépend de la mise en place préalable de la ligne tout entière. Comme il n'y a pas de comportement caractéristique associé à la perception d'un objet rouge, la relation entre les stimuli et les réponses qui sous-tendent la sensation de rouge est complexe et indirecte, et doit être conçue sur un modèle holiste ${ }^{43}$. Par contraste, les liens qui correspondent à une sensation comme la douleur sont si simples et directs que le terme « douleur », compris dans son sens ordinaire, ne peut pas être utilisé pour désigner la cause de la douleur indépendamment de son expression naturelle.

Ce qui vaut pour le langage des sensations vaut également pour la conscience. À cause dela simplicité et de la spontanéité de sa ligne de type, l'expérience douloureuse ne se réduit pas à la perception confuse d'une stimulation nocive. Au contraire, la douleur est une entité essentiellement bifaciale : ce qui est présenté dans l'expérience douloureuse ne peut pas être identifiéindépendamment de la stimulation nocive et de la réponse spontanée - ou au minimum de la tendance à répondre d'une certaine manière lorsque la ligne de type n'est pas effectivement exemplifiée. De même que le langage ne peut pas intervenir entre la douleur et son expression naturelle, la conscience corporelle ne peut pas dissocier les deux moitiés de la ligne de type sur laquelle elle est (normalement) fondée. Lorsquej'ai mal à l'épaule, la condition douloureuse dans laquelle se trouve une partie de mon corps m'apparaît comme simple dans la mesure où je ne peux pas clairement séparer, sur la seule base de mon expérience, la contribution de la stimulation nocive de celle de la réponse spontanée ${ }^{44}$.

43. Voir Pears, La pensée-Wittgenstein, p. 300.

44. Je ne veux pas dire que les sensations corporelles soient les seules à exemplifier ce phénomène. Les « qualités » d'orientation (responsables du fait que je perçois un objet en haut, à droite, au fond, et ainsi de suite, dans mon champ perceptif) ressemblent aux sensations corporelles par la simplicité de leurs lignes de types. Il est intéressant de remarquer que les qualités d'orientation ne sont pas entièrement « externes » : le monde perçu n'est pas intrinsèquement orienté. Plutôt que d'introduire des modes de présentation perceptifs distincts des qualités de la scène perçue, on pourrait considérer les qualités d'orientation comme étant en partie constituées de réponses spontanées. Lorsque je perçois un objet à gauche, je ne perçois pas seulement cet objet, mais également ma propre tendance à tourner les yeux ou la tête vers la gauche (par exemple). 
Selon la suggestion qui vient d'être émise, l'objet intentionnel del'expérience de la douleur est plus qu'un simple dommage physique ; il inclut, ou du moins survient sur les réponses naturellement associées à celui-ci. Cette suggestion est non seulement plausible sur le plan conceptuel ; elle est également compatible avec les données phénoménologiques. Schilder a admirablement bien décrit ce trait fondamental dela douleur et ses conséquences au niveau de l'image du corps :

II semble que toute douleur en un point de la surface du corps renforce le modèle postural en ce point, le distorde, avec des impressions d'enflure ${ }^{45}$.

La partie du corps sur laquelle la douleur est ressentie draine toute l'attention. [...] M ais, en même temps, la partie douloureuse est isolée, quelque chose la pousse hors de l'image du corps ${ }^{46}$.

Il est évident, à partir de cette description, que si l'expérience de la douleur est conçue comme une forme de perception objective, ce qui est perçu n'est pas un simple dommage physique. Le sujet qui souffre perçoit également et simultanément la réponse de son propre corps aux stimuli nocifs.

\section{La réflexivité de la conscience objective interne}

On remarquera à juste titre que la suggestion qui vient d'être émise n'explique en rien la différence pertinente entre la conscience externe et la conscience corporelle. On peut admettre que le même objet intentionnel - une qualité qui dépend de manière constitutive de relations entre des stimuli physiques et des réponses spontanées - est en principe accessible aux deux formes de conscience. II est possible que la même chose me soit présentée à la conscience lorsque je ressens la douleur et lorsque je la vois chez autrui (ou chez moi-même dans un miroir). Je vais tenter de montrer que cette suggestion contient pourtant in nucce des éléments qui nous permettent de rendre compte de la spécificité de la conscience corporelle.

N ous avons vu que la TM S rend difficilement compte du lien intuitif entre la douleur et l'espace corporel. Considérons à présent une autre intuition ordinaire que nous avons sur la douleur. II semble que la notion d'une douleur non ressentie soit incohérente : la douleur, contrairement au dommage physique, n'existe que par l'expérience que nous en avons. Pour la douleur, esse est sentiri. Certains matérialistes considèrent que le terme « douleur » ne désigne pas l'objet intentionnel de la douleur, et donc que cette intuition est compatible avec la TM S ${ }^{47}$. À mon avis, ils se voilent la face devant un fait incontestable : la

45. Schilder, Paul, L'image du corps, Paris, Gallimard, 1968, p. 121.

46. Ibid., p. 125.

47. Voir Tye, Ten Problems of Consciousness, pour qui la douleur est en fait l'expérience de la douleur. $D$ 'autres philosophes pensent que le concept de douleur est incohérent (D ennett, «W Wy You Can't M ake a Computer that Feels Pain ») ou équivoque (Pitcher, «Pain Perception »; Lycan, Consciousness and Experience). 
douleur que je localise dans mon bras n'existe que parce que je la ressens ; elle ne résiste pas à l'anesthésie, et donc ne peut pas être un simple dommage physique.

Shoemaker fait observer qu' « au moins certaines formes de "comportement lié à la douleur" (pain behavior) - prendre de l'aspirine et appeler le médecin en sont de bons exemples - ne sont intelligibles en tant que comportement lié à la douleur que si l'on part du principe que le sujet est conscient (aware) de la douleur ${ }^{48}$ ». Selon lui, il est impossible d'être « aveugle à soimême » (self-blind) lorsque l'on a une douleur. L'existence de la douleur entraîne celle d'une expérience, même inattentive, qui porte sur elle.

L'observation de Shoemaker peut être transposée à l'ensemble des sensations corporelles. La forme générale de l'expérience corporelle est la suivante :

Expérience (cette partie corporelle est F)

Le prédicat $F$ (par exemple, " avoir mal ») désigne une condition dans laquelle mon corps m'est présenté comme se trouvant. M ême si cette condition m'apparaît typiquement comme simple, c'est un fait que mon corps ne peut s'y trouver qu'en présence de l'expérience dont elle est l'objet. L'expérience corporelle est essentiellement réflexive, et c'est parce qu'elle est réflexive que son objet intentionnel lui est lié de manière constitutive.

J'ai affirmé que la douleur, considérée commel'objet intentionnel de la conscience corporelle, survient sur des relations naturelles entre des stimuli nocifs et des réponses spontanées de rejet. Si ces réponses se réduisaient à des gestes réflexes ou à des grimaces, la douleur ne dépendrait sans doute pas d'une expérience consciente. $M$ ais elle serait également très éloignée de ce que nous appel ons « douleur ${ }^{49}$ ». La douleur humaine (comme peut-êtrecertaines formes de douleur animale) a ceci de particulier que parmi les réponses qui la définissent, il y a précisément son entrée dans le champ de la conscience, qui en fait un objet susceptible d'attirer l'attention du sujet.

Del'impossibilité d'être aveugle à sa propre douleur, Shoemaker conclut quel'expérience de la douleur n'est pas une forme de perception objective. Elle serait plutôt une forme non perceptive d'introspection. Je pense que Shoemaker sous-estime la distinction entre « introspecter » une pensée ou une expé rience et prendre conscience d'une sensation corporelle. Dans le premier cas, Shoemaker pourrait avoir raison lorsqu'il affirme que « la réalité connue et la faculté de la connaître sont pour ainsi dire faites l'une pour l'autre - l'une ne pourrait pas être ce qu'elle est sans l'autre ${ }^{50} »$. L'introspection n'est pas une forme de perception objective, avant tout parce que la notion d'illusion introspective est inintelligible. II en va autrement de la conscience d'une sensation corporelle, à laquelle les traits présentés au commencement s'appliquent

48. Shoemaker, The First-Person Perspective, p. 228.

49. Ibid., p. 229.

50. Ibid., p. 245. 
clairement : elle est localisée, elle peut attirer l'attention et, contrairement à la pensée introspective, elle peut être illusoire sous certains aspects. Plutôt que d'assimiler la conscience corporelle à l'introspection (ou inversement), je préfère la considérer comme une forme de conscience intermédiaire entre la perception externe, avec laquelle ellepartage des traits qui définissent l'expérience objective, et l'introspection, qui n'a aucun de ces traits.

Selon mon hypothèse, que je présente ici comme une piste de recherche, c'est la réflexivité qui distingue (au moins partiellement) la conscience corporelle de la conscience externe. La douleur réelle est un état corporel essentiellement psychophysique; elle suppose des stimuli physiques, mais elle engage de manière constitutive une expérience consciente. Lorsque je perçois la douleur "à l'extérieur », par exemple chez autrui, je perçois un état de choses complexe : un dommage ou un dérangement corporel et l'expérience du sujet focalisée sur le lieu douloureux. M on acte de perception et l'expérience que je perçois sont des existences distinctes, car il n'y a qu'une relation causale entre la douleur et l'expérience que j'en ai. Par contraste, lorsque je ressens la douleur dans mon corps propre, un objet intentionnel du même type se présente à ma conscience, mais celle-ci est littéralement identique à l'expérience présentée ; il y a donc une relation constitutive entre la douleur et l'expérience que j'en ai. L'hypothèse est que cette différence contribue à expliquer pourquoi c'est seulement dans ce deuxième cas que je prends conscience de la douleur « de l'intérieur ${ }^{51} »$.

De ce point de vue, l'intuition sel on laquelle la douleur n'existe que par l'expérience que j'en ai est indissociable de l'intuition selon laquelle elle est nécessairement liée au corps propre. La douleur dépend ontologiquement de mon expérience parce que celle-ci est l'une des réponses qui la constitue. Aucun état du corps d'autrui ne pourrait avoir cette propriété. Si je suis réellement en présence d'une réponse consciente spontanée à des stimuli nocifs, et si je perçois cet état de choses « de l'intérieur », il ne peut concerner que mon propre corps, puisqu'il concerne ma propre expérience. Le critère (D) énoncé plus haut est donc essentiellement correct.

Une telle explication ne peut être considérée que comme une ébauche. Par exemple, il faudrait montrer plus en détail la manière dont la réflexivité de l'expérience corporelle est apparente au sujet, comment elle participe de l'effet que cela fait d'avoir une sensation corporelle. Je considère néanmoins qu'elle est plus prometteuse que la TM S, qui fait del'objet intentionnel del'expérience

51. On objectera que cette hypothèse ne vaut que si la conscience externe n'est pas (normalement) réflexive, alors que Searle, par exemple, a montré qu'elle l'était aussi (voir Searle, John, L'intentionnalité, traduction C. Pichevin, Paris, M inuit, 1987, ch. 2). L'interprétation de la théorie sui-référentielle de Searle est controversée (voir la réponse de Searle à Tyler Burge et à John M cD owell dans Lepore, Ernest, et van Gulick, Robert, dir., John Searle and $\mathrm{H}$ is Critics, Oxford, Blackwell, 1991), mais s'il veut dire que le contenu apparent de l'expérience visuelle inclut cette même expérience, il me semble que sa position est critiquable au moins sur des bases phénoménologiques. 
de la douleur un simple dommage physique. Cette théorie est incapable de satisfaire aux intuitions récalcitrantes que nous avons sur la douleur et sur la conscience corporelle en général. L'explication qui vient d'être esquissée, en revanche, est en principe compatible avec le matérialisme, et a le mérite de prendre nos intuitions au sérieux. Si elle est sur la bonne voie, le matérialisme ne doit pas avoir peur des qualia corporels, du moins s'il ne craint pas ceux de la perception en général. 